\title{
SIMPLEXES IN RIEMANNIAN MANIFOLDS
}

\author{
B. V. DEKSTER
}

(Communicated by Jonathan M. Rosenberg)

\begin{abstract}
Existence of a simplex with prescribed edge lengths in Euclidean, spherical, and hyperbolic spaces was studied recently. A simple sufficient condition of this existence is, roughly speaking, that the lengths do not differ too much. We extend these results to Riemannian $n$-manifolds $M^{n}$. More precisely we consider $m+1$ points $p_{0}, p_{1}, \ldots, p_{m}$ in $M^{n}, m \leq n$, with prescribed mutual distances $l_{i j}$ and establish a condition on the matrix $\left(l_{i j}\right)$ under which the points $p_{i}$ can be selected as freely as in $R^{n}: p_{0}$ is a prescribed point, the shortest path $p_{0} p_{1}$ has a prescribed direction at $p_{0}$, the triangle $p_{0} p_{1} p_{2}$ determines a prescribed 2-dimensional direction at $p_{0}$, and so on.
\end{abstract}

\section{BASIC DEFINITIONS AND THE THEOREM}

Existence of a simplex with prescribed edge lengths in Euclidean, spherical, and hyperbolic spaces was studied in [3]. A simple sufficient condition of this existence established there is, roughly speaking, that the edge lengths do not differ too much, see [3, Theorem 2]. We deal here with $m+1$ points $p_{0}, p_{1}, \ldots, p_{m}$ in a Riemannian $n$-manifold $M^{n}, m \leq n$, with prescribed mutual distances $l_{i j}$ and establish a condition on the matrix $\left(l_{i j}\right)$ under which the points $p_{i}$ can be selected as freely as in $R^{n}: p_{0}$ is a prescribed point, the shortest path $p_{0} p_{1}$ has a prescribed direction at $p_{0}$, the triangle $p_{0} p_{1} p_{2}$ determines a prescribed 2dimensional direction at $p_{0}$, and so on. Our result however does not guarantee uniqueness of the points $p_{i}$ (see more on that at the ends of parts $\mathrm{A}$ and $\mathrm{I}$ of $\S 3)$. Note that the desired points $p_{i}$ may not exist even though all the distances $l_{i j}$ are equal and the manifold $M^{n}$ is complete, noncompact, and expanding in the following sense: there exists a point $w \in M^{n}$ and a constant $c>0$ such that for any triangle $a w b$ with $w a=w b$, one has $a b>c \cdot w a \cdot \angle a w b$ where $\angle$ means angle. An appropriate example for four points in $M^{3}$ can be constructed as follows. Let $M^{2}$ be a narrow right circular cone. Its vertex $v$ can be smoothed out later for regularity. Put $M^{3}=M^{2} \times R$. One can check that $M^{3}$ is expanding if the point $(v, 0)$ is chosen as the point $w$. Prescribe $l_{i j}=1$.

Received by the editors November 19, 1990 and, in revised form, December 3, 1991; presented on March 13, 1991, at the 865th Meeting of the AMS at Tampa, Florida.

1991 Mathematics Subject Classification. Primary 53C42, 53C99; Secondary 51K99, 52A99.

Key words and phrases. Simplexes, mutual distances between points, comparison theorems for triangles.

Supported by a Canadian NSERC Grant. 
Select points $q_{0}, q_{1}$, and $q_{2} \in M^{2}$ on a circumference centered at $v$ such that their mutual distances in $M^{2}$ all are unit. Now put $p_{0}=\left(q_{0}, 0\right), p_{1}=\left(q_{1}, 0\right)$, and $p_{2}=\left(q_{2}, 0\right)$. Obviously $l_{01}=l_{02}=l_{12}=1$. By symmetry, the last point $p_{3}$ should be of the form $(v, h), h \in R$. Then $l_{30}=l_{31}=l_{32}=\left(r^{2}-h^{2}\right)^{1 / 2}$ where $r$ is the radius of the above circumference. When the cone $M^{2}$ is sufficiently narrow, one has $r>1$. Then $l_{30}=l_{31}=l_{32}>1$ and hence the desired point $p_{3}$ does not exist when $p_{0}, p_{1}$, and $p_{2}$ are selected as above.

There is another subtle difference in this area between $M^{n}$ and Euclidean, hyperbolic, or spherical $n$-space $X_{k}^{n}$ of curvature $k$. Consider, say, a tetrahedron in $X_{k}^{n}$. If the directions of the three edges coming from its vertex are coplanar then the same is true of each other vertex of the tetrahedron. This is not so in $M^{n}$ even for small tetrahedra.

By $k$-plane, we will mean $X_{k}^{2}$. The sphere $X_{1}^{n}$ will often be denoted by $S^{n}$.

The notation $x y$ will be used for a geodesic with ends $x, y$ for its length and for the distance between $x$ and $y$. The meaning will be specified in cases of possible confusions.

A set $C \subset M^{n}$ is called convex if for each two points in $C$ there exists a unique shortest path in $M^{n}$ connecting these points and this path (which is a geodesic) belongs to $C$.

Let $\left(x_{i j}\right)$ be a matrix with $x_{i i}=0, x_{i j}=x_{j i}>0, i, j=r, r+1, \ldots, r+s$. (We will encounter cases $r=0$ and $r=1$.) Such a matrix will be called allowable. Let $q_{r}, q_{r+1}, \ldots, q_{r+s}$ be $s+1$ points in a metric space $Y$ with the mutual distances $q_{i} q_{j}=x_{i j}$. The set of these $s+1$ points will be called a realization of $\left(x_{i j}\right)$ in $Y$ and often written down as $q_{r} q_{r+1} \cdots q_{r+s}$. Suppose $Y=X_{k}^{n}$ with $n \geq s$. In case $k>0$, assume also that the points $q_{i}$ lie in an open semisphere of $X_{k}^{n}$. If their convex hull is a nondegenerate $s$-simplex then we say that the matrix $\left(x_{i j}\right)$ and its realization are nondegenerate in $X_{k}^{n}$.

Let $M^{n}, n \geq 2$, be a regular Riemannian manifold and let $e_{1}, e_{2}, \ldots, e_{m}$, $2 \leq m \leq n$, be pairwise orthogonal unit vectors at a point $p \in M^{n}$. The set $\left\{e_{1}, e_{2}, \ldots, e_{m}\right\}$ will be called a frame at $p$. Suppose that an allowable matrix $\left(l_{i j}\right), i, j=0,1, \ldots, m$, has a realization $p_{0} p_{1} \cdots p_{m}$ in $M^{n}$ such that, for each pair $p_{i}, p_{j}$, the manifold $M^{n}$ contains a unique shortest geodesic $p_{i} p_{j}$ of the length $l_{i j}$ and the following conditions hold.

(0) $p_{0}=p$.

(1) The direction of the segment $p_{0} p_{1}$ is $e_{1}$.

(2) The direction of the segment $p_{0} p_{2}$ is coplanar with $e_{1}$ and $e_{2}$ and forms with $e_{2}$ an angle $<\pi / 2$.

(3) The direction of the segment $p_{0} p_{3}$ is coplanar with $e_{1}, e_{2}$, and $e_{3}$ and forms with $e_{3}$ an angle $<\pi / 2$.

(m) The direction of the segment $p_{0} p_{m}$ is coplanar with $e_{1}, e_{2}, \ldots, e_{m}$ and forms with $e_{m}$ an angle $<\pi / 2$.

We will say then that the realization $p_{0} p_{1} \cdots p_{m}$ of $\left(l_{i j}\right)$ fits the frame $\left\{e_{1}, e_{2}\right.$, $\left.\ldots, e_{m}\right\}$ at $p$.

Theorem. Let $M^{n}, n \geq 2$, be a regular Riemannian $n$-manifold, not necessarily complete. Let $p \in M^{n}, r>0$ be less than or equal to the convexity radius at $p$ (see [4, §5.2] for the definition), let $k^{\prime}$ and $k^{\prime \prime}$ be finite lower and upper bounds of the sectional curvature in the r-neighbourhood $N_{r}(p)$ of $p$, and let 
$\left\{e_{1}, e_{2}, \ldots, e_{m}\right\}, 2 \leq m \leq n$, be a frame at $p$. Suppose that an allowable matrix $\left(l_{i j}\right), i, j=0,1, \ldots, m$, satisfies the following conditions:

(i) $l_{0 i}<r, i=1,2, \ldots, m$.

(ii) For each pair of distinct $i$ and $j$ different from 0 , there exists a nondegenerate triangle on the $k^{\prime}$-plane ( $k^{\prime \prime}$-plane) with side lengths $l_{0 i}, l_{0 j}$, and $l_{i j}$.

$$
\text { (Thus its perimeter is }<2 \pi / \sqrt{k^{\prime \prime}} \text { if } k^{\prime \prime}>0 \text {.) }
$$

(iii) With $\alpha_{i j}^{\prime}\left(\alpha_{i j}^{\prime \prime}\right)$ being the angle of that triangle opposite to the side of the length $l_{i j}$, each allowable matrix $\left(\alpha_{i j}\right)$ satisfying

$$
\alpha_{i j}^{\prime} \leq \alpha_{i j} \leq \alpha_{i j}^{\prime \prime}, \quad i, j=1,2, \ldots, m,
$$

has a nondegenerate realization $a_{1} a_{2} \cdots a_{m}$ in $S^{n-1}$.

Then the matrix $\left(l_{i j}\right)$ has a realization $p_{0} p_{1} \cdots p_{m}$ in $M^{n}$ which fits the frame $\left\{e_{1}, e_{2}, \ldots, e_{m}\right\}$. Moreover, any realization $p_{0} p_{1} \cdots p_{k}, k<m$, of the matrix $\left(l_{i j}\right)$ with $i, j=0,1, \ldots, k$ fitting the frame $\left\{e_{1}, e_{2}, \ldots, e_{k}\right\}$ (we do not know if such a realization is unique) can be augmented by points $p_{k+1}, p_{k+2}, \ldots, p_{m}$ such that the resulting set $p_{1} p_{2} \cdots p_{m}$ is a realization of the original matrix $\left(l_{i j}\right)$ fitting the frame $\left\{e_{1}, e_{2}, \ldots, e_{m}\right\}$.

\section{SOME RELATED QUESTIONS}

Remark 1. Condition (ii) of the theorem is not easy to check directly. Theorem 2 in [3] yields a simple sufficient condition for (iii) to hold. Take some

$$
\alpha \geq \max _{i, j} \alpha_{i j}^{\prime \prime}
$$

and suppose that

$$
\alpha \leq 2 \arcsin \sqrt{\frac{m}{2(m-1)}} .
$$

Then, by [3, Theorem 2], a quantity

$$
\lambda=\lambda(m-1, \alpha) \in(0, \alpha)
$$

is determined identically by the equation

$$
\sin \lambda=\sin \alpha\left[1-f(m-1)\left(\cos ^{2} R\right) / \cos ^{2}(\alpha / 2)\right]^{1 / 2}
$$

where

$$
f(m-1)= \begin{cases}2 / m & \text { if } m \text { is even }, \\ 2 m /(m-1)(m+1) & \text { if } m \text { is odd }\end{cases}
$$

and

$$
\sin R=\sqrt{2(m-1) / m} \sin (\alpha / 2) .
$$

(We use $m-1$ as the integer argument to comply with [3].) This $\lambda$ has the property that each allowable $m \times m$ matrix $\left(\alpha_{i j}\right)$ with

$$
\alpha_{i j} \in(\lambda, \alpha], \quad i \neq j,
$$

is nondegenerate in $S^{m-1}$. Thus if $\min _{i, j} \alpha_{i j}^{\prime}>\lambda=\lambda(m-1, \alpha)$ then (iii) holds. 
Remark 2. A better though less convenient method to check condition (iii) arises from Theorem 1 in [3]. Put

$$
s_{i j}=\cos \alpha_{i j}-\cos \alpha_{i m} \cos \alpha_{j m}, \quad i, j=1,2, \ldots, m-1 .
$$

Theorem 1 in [3] implies that the $m \times m$ matrix $\left(\alpha_{i j}\right)$ of our theorem has a nondegenerate realization in $S^{m-1}$ if and only if the $(m-1) \times(m-1)$ matrix $S=\left(s_{i j}\right)$ has a positive spectrum. To establish this property of $S=S\left(\alpha_{i j}\right)$ for each combination of the $m(m-1) / 2$ arguments $\alpha_{i j}$ in the domain $D: a_{i j}^{\prime} \leq$ $\alpha_{i j} \leq \alpha_{i j}^{\prime \prime}$, it is enough to establish this property for one such combination and then make sure that $\operatorname{det} S\left(\alpha_{i j}\right)$ stays positive in $D$.

Remark 3. Suppose the sectional curvature is constant in $N_{r}(p)$. Then one can take $k^{\prime}=k^{\prime \prime}=k$. The matrix $\left(\alpha_{i j}\right)$ is now unique. Its realizability can be checked with the help of Theorems 1 and 2 in [3]. Our theorem means now that each allowable matrix $\left(l_{i j}\right)$ is freely realizable in $N_{r}(p)$ with 0 th vertex at $p$ (see the next remark) if and only if it satisfies (i), (ii), and (iii). (Considering necessity of (iii), one should notice that fitting the frame $\left\{e_{1}, e_{2}, \ldots, e_{m}\right\}$ implies nondegeneracy of the realization $p_{0} p_{1} \cdots p_{m}$ in $N_{r}(p)$, which in turn implies nondegeneracy of the realization $a_{1} \cdots a_{m}$ in $S^{n-1}$.) In this form, the theorem can be applied to spaces of constant curvature more general than $X_{k}^{n}$.

Let, for instance, $M^{3}=S^{1} \times R^{2}$. Then the convexity radius $R(p)=\pi / 2$ for any $p \in M^{3}$. Take $r=\pi / 2$. Consider the matrix

$$
L=\left(\begin{array}{ccc}
0 & l_{01} & l_{02} \\
l_{10} & 0 & l_{12} \\
l_{20} & l_{21} & 0
\end{array}\right)=\left(\begin{array}{ccc}
0 & 1.58 & 1.58 \\
1.58 & 0 & 3.15 \\
1.58 & 3.15 & 0
\end{array}\right)
$$

where $1.58>\pi / 2$ and $3.15>\pi$. This matrix has realizations in $M^{3}$, say, those located in $R^{2}$. The theorem, however, does not guarantee existence of any realization of $L$ since $l_{01}>r$ in violation of (i). Importance of (i) becomes clear if one notices that $L$ has no realization in $S^{1} \times R^{1} \subset M^{3}$ which would be symmetric about $R^{1}$. At the same time, replacing 1.58 and 3.15 by 1.57 and 3.13 , one gets a matrix freely realizable in $M^{3}$ (see Remark 5 for an exact definition) according to the theorem.

Remark 4. Let $K$ be a nonempty set in $M^{n}$ and let $\left(l_{i j}\right)$ be an allowable matrix. If for any $p \in K$ and any frame $\left\{e_{1}, e_{2}, \ldots, e_{m}\right\}, 2 \leq m \leq n$, at $p$ the matrix $\left(l_{i j}\right)$ has a realization in $M^{n}$ fitting this frame, we will say that $\left(l_{i j}\right)$ is freely realizable in $M^{n}$ with 0 th vertex in $K$. The theorem gives a simple sufficient condition of such realizability. Suppose that $\inf _{p \in K} R(p)>0$ where $R(p)$ is the radius of convexity. Take a positive $r \leq \inf _{p \in K} R(p)$ and let $k^{\prime}, k^{\prime \prime}$ be finite lower and upper bounds of sectional curvature in the $r$-neighbourhood of $K$. Suppose that conditions (i), (ii), and (iii) hold with these $r, k^{\prime}, k^{\prime \prime}$. The theorem implies then that $\left(l_{i j}\right)$ is freely realizable in $M^{n}$ with 0 th vertex in $K$.

Remark 5. Let $P$ be a permutation on $\{0,1, \ldots, m\}$. Put $l_{i j}^{P}=l_{P(i) P(j)}$, $i, j=0,1, \ldots, m$. If the matrix $\left(l_{i j}^{P}\right)$ is freely realizable in $M^{n}$ with 0 th vertex in $K$ for any $P$, we will say that the original matrix $\left(l_{i j}\right)$ is freely realizable in $M^{n}$ with a vertex in $K$. If $K=M^{n}$ here, we will say that $\left(l_{i j}\right)$ is freely realizable in $M^{n}$. (In this case, $M^{n}$ of course should be complete.) 
Free realizability with 0 th vertex in $K$ does not imply free realizability with a vertex in $K$. In the rest of this remark, we assume that $r, k^{\prime}$, and $k^{\prime \prime}$ are as in the preceding remark. Note that conditions (i), (ii), and (iii) can hold for $\left(l_{i j}\right)$ but fail for $\left(l_{i j}^{P}\right)$. Of course, if (i), (ii), and (iii) hold for each matrix $\left(l_{i j}^{P}\right)$ then $\left(l_{i j}\right)$ is freely realizable with a vertex in $K$.

A natural question to ask in this connection is as follows. Suppose that $\left(l_{i j}\right)$ is freely realizable in $M^{n}$ with 0 th vertex in $K$. Put

$$
\begin{aligned}
& \tilde{l}=\max _{i} l_{0 i} ; \\
& \widetilde{K}= \begin{cases}\{x \in K \mid \rho(x, \partial K) \geq \tilde{l}\} & \text { if } \partial K \neq \varnothing, \\
M^{n} & \text { if } \partial K=\varnothing\left(\text { then } K=M^{n}\right) .\end{cases}
\end{aligned}
$$

Suppose $\tilde{K} \neq \varnothing$. It is guaranteed now that each realization $p_{0} p_{1} \cdots p_{m}$ of $\left(l_{i j}\right)$ with at least one vertex, say $p_{1}$, in $\widetilde{K}$ has $p_{0} \in K$. Let $F=\left\{f_{1}, f_{2}, \ldots, f_{m}\right\}$ be a frame at the point $p_{1} \in \widetilde{K}$. Is it possible to select $p_{0} \in K$ and a frame $\left\{e_{1}, e_{2}, \ldots, e_{m}\right\}$ at $p_{0}$ such that the realization $p_{0} p_{1} \cdots p_{m}$ would fit both frames? In other words, is $\left(l_{i j}\right)$ freely realizable in $M^{n}$ with a vertex in $\widetilde{K}$ ? We do not know the answer.

Remark 6. Let $K, r>0, k^{\prime}$, and $k^{\prime \prime}$ be as in Remark 4. Let $L=\left(l_{i j}\right), i, j=$ $0,1, \ldots, m$, be the matrix of the edge lengths of a nondegenerate Euclidean $m$ simplex. Put $L(\varepsilon)=\left(\varepsilon l_{i j}\right), \varepsilon>0$. Then $L(\varepsilon)$ is freely realizable in $M^{n}$ with 0 th vertex in $K$ for sufficiently small $\varepsilon$. Indeed, together with the angles $\alpha_{i j}^{\prime}=$ $\alpha_{i j}^{\prime}(\varepsilon)$ and $a_{i j}^{\prime \prime}=\alpha_{i j}^{\prime \prime}(\varepsilon)$ on $k^{\prime}$ - and $k^{\prime \prime}$-planes for the matrix $L(\varepsilon)$, consider also the appropriate angles $\alpha_{i j}^{0}$ on Euclidean plane. (They do not depend on $\varepsilon$.) The matrix $\left(\alpha_{i j}^{0}\right)$ is nondegenerate in $S^{m-1}$ since $L$ is nondegenerate in $R^{m}$. Obviously, $\alpha_{i j}^{\prime} \rightarrow \alpha_{i j}^{0}, \alpha_{i j}^{\prime \prime} \rightarrow \alpha_{i j}^{0}$ as $\varepsilon \rightarrow 0$. Then, for sufficiently small $\varepsilon$, any matrix $\left(\alpha_{i j}\right)$ with $\alpha_{i j} \in\left[\alpha_{i j}^{\prime}(\varepsilon), \alpha_{i j}^{\prime \prime}(\varepsilon)\right]$ is arbitrarily close to the nondegenerate $\left(\alpha_{i j}^{0}\right)$. Since nondegenerate matrices form an open set in the appropriate matrix space (see [3, Corollary of Theorem 1]), these matrices $\left(\alpha_{i j}\right)$ are nondegenerate. Now the theorem implies that $L(\varepsilon)$ is freely realizable in $M^{n}$ with 0 th vertex in $K$.

Applying this observation to each permuted matrix $l_{i j}^{P}$ (see Remark 5), one will see also that $L(\varepsilon)$ is freely realizable in $M^{n}$ with a vertex in $K$ when $\varepsilon$ is sufficiently small.

Remark 7. Note finally that the theorem does not assume triangle inequalities involving $l_{i j}, l_{i k}, l_{j k}$ in which the index 0 does not appear among $i, j, k$. Those triangle inequalities follow from the theorem, i.e., from realizability of the matrix $\left(l_{i j}\right)$.

\section{Proof of the theorem}

A. One may consider only the case $k=m-1$ since points can be added one at a time. We use induction by $m$. If $m=2$, one obviously can select $p_{0}$ and $p_{1}$ fitting the frame $\left\{e_{1}\right\}$. (This selection is unique in this particular case.) Let $p(\phi)$ be such that the length $p_{0} p(\phi)=l_{02}$, and the direction of the segment $p_{0} p(\phi)$ is coplanar with $e_{1}$ and $e_{2}$ and forms an angle $\phi \in[0, \pi]$ with $e_{1}$ and an angle $\leq \pi / 2$ with $e_{2}$. The distance $p_{1} p(\phi)$ changes continuously with $\phi$ 
from $p_{1} p(0)=\left|l_{01}-l_{02}\right| \leq l_{12}$ to $p_{1} p(\pi)=l_{01}+l_{02} \geq l_{12}$. Then $p_{1} p\left(\phi^{*}\right)=l_{12}$ for some $\phi^{*} \in[0, \pi]$. If $\phi^{*}=\pi$ then $l_{12}=l_{01}+l_{02}$, which is impossible for the nondegenerate triangles mentioned in (ii). Thus $\phi^{*} \neq \pi$. If $\phi^{*}=0$, then either $l_{01}-l_{02}=l_{12}$ or $l_{02}-l_{01}=l_{12}$, which is also impossible due to (ii). Hence $\phi^{*} \in(0, \pi)$ and the point $p_{2}=p\left(\phi^{*}\right)$ is a desirable one. (We do not know if $\phi^{*}$ and $p_{2}$ are unique.) Thus the theorem, including the statement on augmentation, holds for $m=2$.

B. Suppose now that the theorem holds for $m-1 \geq 2$ in place of $m$. Along with the matrix $L=\left(l_{i j}\right), i, j=0,1, \ldots, m$, we will consider three other matrices: $L_{m}$ with is obtained from $L$ by deleting its $m$ th, i.e., the last, row and column; $L_{m-1}$ obtained from $L$ by deleting its $(m-1)$ st row and column; and $L_{m-1 m}$ obtained from $L$ by deleting its last two rows and last two columns. Similarly, we introduce three modifications, $A_{m}, A_{m-1}$, and $A_{m-1 m}$, of a matrix $A=\left(\alpha_{i j}\right), i, j=1,2, \ldots, m$. Note that since $A$ has a nondegenerate realization in $S^{n-1}$ for any choice of its elements $\alpha_{i j} \in\left[\alpha_{i j}^{\prime}, \alpha_{i j}^{\prime \prime}\right]$, the same is true of $A_{m}, A_{m-1}$, and $A_{m-1 m}$. By our induction assumption, there exists a realization $p_{0} p_{1} \cdots p_{m-1}$ of $L_{m}$ fitting the frame $\left\{e_{1}, e_{2}, \ldots, e_{m-1}\right\}$. For $\phi \in[0, \pi]$, denote by $e(\phi)$ the unit vector coplanar to $e_{m-1}$ and $e_{m}$ forming an angle $\phi$ with $e_{m-1}$ and an angle $\leq \pi / 2$ with $e_{m}$. Obviously the part $p_{0} p_{1} \cdots p_{m-2}$ of the last realization is a realization of $L_{m-1 m}$. By our induction assumption, this realization $p_{0} p_{1} \cdots p_{m-2}$ can be augmented by a point $p(\phi)$ such that the resulting set $p_{0} p_{1} \cdots p_{m-2} p(\phi)$ is a realization of $L_{m-1}$ fitting the frame $\left\{e_{1}, e_{2}, \ldots\right.$, $\left.e_{m-2}, e(\phi)\right\}$. Denote by $a_{1}, \ldots, a_{m-1}, a(\phi)$ the directions of the segments $p_{0} p_{1}, \ldots, p_{0} p_{m-1}, p_{0} p(\phi)$ at $p_{0}$. We now specify the entries $a_{i j}$ of the matrix $A$ above as follows. We assume $a_{i j}$ to be the distance $a_{i} a_{j}$ on the sphere $S^{n-1}$ of directions at $p_{0}$ for $i, j \leq m-1$, i.e.,

$$
\alpha_{i j}=a_{i} a_{j}=\angle p_{i} p_{0} p_{j}, \quad i, j=1,2, \ldots, m-1 \text {. }
$$

We put

$$
\alpha_{i m}=\alpha_{m i}=a_{i} a(\phi), \quad i=1,2, \ldots, m-1, \quad \alpha_{m m}=0 .
$$

Thus $a_{1} \cdots a_{m-1} a(\phi)$ is now a realization of the matrix $A$ in $S^{n-1}$ while $a_{1} \cdots a_{m-1}, \quad a_{1} \cdots a_{m-2} a(\phi)$, and $a_{1} \cdots a_{m-2}$ are realizations of $A_{m}, A_{m-1}$, and $A_{m-1 m}$. Note that in case $M^{n}=X_{k}^{n}$, the entries $\alpha_{i m}$ do not depend on $\phi$ except for $\alpha_{m-1 m}$.

C. We make now an important reference to comparison theorems for triangles by Alexandrow and Toponogov. That will be the only substantial reference to Riemannian Geometry in this paper. Since $l_{0 i}<r$, all our points $p_{0}, p_{1}, \ldots, p_{m-1}, p(\phi)$ and the segments between them lie in $N_{r}\left(p_{0}\right)$. According to $[2, \S 6.4 .2$, Theorem and Remark 3], Toponogov's Theorem can be stated as follows.

Theorem (V. A. Toponogov). Let $C$ be a convex set in $M^{n}, n \geq 2$, and $k^{\prime}$ be a lower bound of the sectional curvature at points of $C$. Then for any triangle made of shortest paths in $C$ there exists a triangle in the $k^{\prime}$-plane with the same side lengths such that the angles $\alpha, \beta, \gamma$ of the triangle in $C$ and the corresponding angles $\alpha^{\prime}, \beta^{\prime}, \gamma^{\prime}$ of the triangle in the $k^{\prime}$-plane satisfy

$$
\alpha^{\prime} \leq \alpha, \quad \beta^{\prime} \leq \beta, \quad \gamma^{\prime} \leq \gamma .
$$


Since $N_{r}\left(p_{0}\right)$ is convex, the theorem applies to it and yields

$$
\begin{gathered}
\alpha_{i j}^{\prime} \leq \alpha_{i j}, \quad i, j=1,2, \ldots, m-1 ; \\
\alpha_{i m}^{\prime} \leq \alpha_{i m}=a_{i} a(\phi)=\angle p_{i} p_{0} p(\phi) \text { for } i \leq m-2 \text { and } \phi \in[0, \pi] .
\end{gathered}
$$

The local comparisons of the angles of triangles like those in (13) and (14) were actually understood prior to Toponogov's global results, e.g., by Alexandrow.

Note that $\alpha_{m-1 m}=\angle p_{m-1} p_{0} p(\phi)$ is not involved in either (13) or (14) since, generally speaking, $p_{m-1} p(\phi) \neq l_{m-1 m}$. (We are just working towards the appropriate equality.) Denote by $B$ the closed metric ball centered at $p_{0}$ whose radius is $\max _{1 \leq i \leq m} l_{i 0}$. Since this radius is $<r$, the ball $B$ is convex. According to $[1, \S 1.7 \mathrm{~b})], B$ is a domain of type $R_{k^{\prime \prime}}$ (defined in $[1, \S 1.4]$ ). It follows from [1, the end of $\S 1.6$ and $\S 1.4 \mathrm{c})]$ that, for any triangle in $B$ of perimeter $<2 \pi \sqrt{k^{\prime \prime}}$, if $k^{\prime \prime}>0$, the triangle in the $k^{\prime \prime}$-plane with the same side lengths satisfies

$$
\alpha \leq \alpha^{\prime \prime}, \quad \beta \leq \beta^{\prime \prime}, \quad \gamma \leq \gamma^{\prime \prime}
$$

where $\alpha, \beta, \gamma$ are the angles of the triangle in $B$ and $\alpha^{\prime \prime}, \beta^{\prime \prime}, \gamma^{\prime \prime}$ are the corresponding angles of the triangle on the $k^{\prime \prime}$-plane. Due to (1), the estimate (15) can be applied to the triangles $p_{i} p_{0} p_{j}$ and $p_{i} p_{0} p(\phi)$ resulting in

$$
\begin{gathered}
\alpha_{i j} \leq \alpha_{i j}^{\prime \prime}, \quad i, j=1,2, \ldots, m-1 ; \\
\alpha_{i m}=\angle p_{i} p_{0} p(\phi) \leq \alpha_{i m}^{\prime \prime} \quad \text { for } i \leq m-2 \text { and } \phi \in[0, \pi] .
\end{gathered}
$$

The relations (13), (14), (16), and (17) mean that the off-diagonal elements of the matrices $A_{m}$ and $A_{m-1}$ satisfy condition (2). Therefore their realizations $a_{1} \cdots a_{m-1}$ and $a_{1} \cdots a_{m-2} a(\phi)$ are nondegenerate.

D. It will be convenient to associate with these two realizations the nondegenerate spherical $(m-2)$-simplexes $a_{1} a_{2} \cdots a_{m-1}$ and $a_{1} a_{2} \cdots a_{m-2} a(\phi)$ of which the first one is immovable while the second one varies with $\phi$. The variation, however, is not rotation about the common $(m-3)$-face $a_{1} a_{2} \cdots a_{m-2}$ since the lengths of the edges $a_{i} a(\phi), i \leq m-2$, generally speaking depend on $\phi$ (see (11)) unless $M^{n}=X_{k}^{n}$. In subsections $\mathrm{E}, \mathrm{F}, \mathrm{G}$, and $\mathrm{H}$ we are going to watch the distance $\alpha(\phi)=a_{m-1} a(\phi)$ in $S^{n-1}$ as $\phi$ varies on $[0, \pi]$.

E. Let $S^{m-2} \subset S^{n-1}$ be the sphere determined by $a_{1} a_{2} \cdots a_{m-1}$. Denote by $H_{0}$ and $H_{\pi}$ the two closed semispheres of $S^{m-2}$ whose common boundary is the spere $S^{m-3}$ determined by $a_{1} a_{2} \cdots a_{m-2}$; see Figure 1 on the next page. Since the simplex $a_{1} a_{2} \cdots a_{m-1}$ is nondegenerate, the point $a_{m-1} \notin S^{m-3}$. One may assume that

$$
a_{m-1} \in \text { relint } H_{0}=H_{0} \backslash S^{m-3} \text {. }
$$

Put

$$
\begin{aligned}
& F_{0}=\left\{x \in H_{0} \mid x a_{i} \in\left[\alpha_{i m}^{\prime}, \alpha_{i m}^{\prime \prime}\right], \quad i=1,2, \ldots, m-2\right\} \\
& F_{\pi}=\left\{x \in H_{\pi} \mid x a_{i} \in\left[\alpha_{i m}^{\prime}, \alpha_{i m}^{\prime \prime}\right], \quad i=1,2, \ldots, m-2\right\} .
\end{aligned}
$$

Let us show that

$$
a(0) \in F_{0}, \quad a(\pi) \in F_{\pi} .
$$




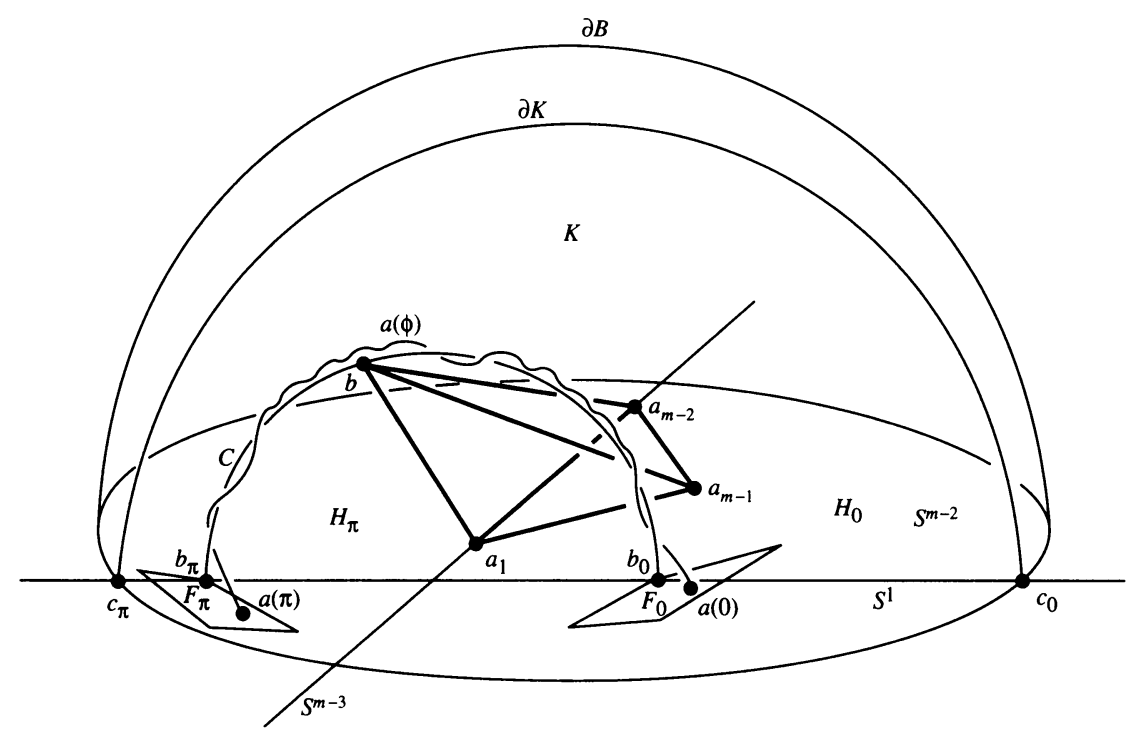

Figure 1

Since $p_{0} p_{1} \cdots p_{m-1}$ and $p_{0} p_{1} \cdots p_{m-2} p(0)$ both fit the same frame $\left\{e_{1}, e_{2}, \ldots\right.$, $\left.e_{m-1}=e(0)\right\}$, the direction $a(0)$ of the segment $p_{0} p(0)$ lies in $H_{0}$ as the point $a_{m-1}$ does according to (18). The distances $a_{i} a(0)=\alpha_{i m}(0), i \leq m-2$ (see (11)), satisfy (2) according to (14) and (17). Thus $a(0) \in F_{0}$. Similarly $a(\pi) \in F_{\pi}$.

F. Denote by $G$ the sperical shell

$$
G=\left\{x \in S^{n-1} \mid \alpha_{m-1 m}^{\prime} \leq x a_{m-1} \leq \alpha_{m-1 m}^{\prime \prime}\right\}
$$

Let us show that

$$
F_{0} \cap G=\varnothing ; \quad F_{\pi} \cap G=\varnothing .
$$

Suppose to the contrary that, say, $F_{0} \cap G \ni z$. Then the mutual distances of the $m$ points $a_{1}, \ldots, a_{m-1}, z$ in $S^{n-1}$ satisfy (2), and hence $a_{1} \cdots a_{m-1} z$ should be a nondegenerate $(m-1)$-simplex. On the other hand, these $m$ points lie in $S^{m-2}$.

G. We prove now that the spherical shell

$$
G \text { separates } F_{0} \text { and } F_{\pi} \text {. }
$$

Suppose to the contrary that, for instance,

$$
F_{0} \cup F_{\pi} \subset B \stackrel{\text { def }}{=}\left\{x \in S^{n-1} \mid x a_{m-1}<\alpha_{m-1 m}^{\prime}\right\} .
$$

Consider the matrix

$$
A^{\prime} \stackrel{\text { def }}{=}\left(\begin{array}{ccccc}
0 & \alpha_{12} & \ldots & \alpha_{1 m-1} & \alpha_{1 m}^{\prime} \\
a_{21} & 0 & \ldots & \alpha_{2 m-1} & \alpha_{2 m}^{\prime} \\
\ldots & \ldots & \ldots & \ldots & \ldots \\
\alpha_{m-11} & \alpha_{m-12} & \ldots & 0 & \alpha_{m-1 m}^{\prime} \\
\alpha_{m 1}^{\prime} & \alpha_{m 2}^{\prime} & \ldots & \alpha_{m m-1}^{\prime} & 0
\end{array}\right)
$$


where $\alpha_{i j}$ are defined by (10). Due to (13) and (16), condition (2) holds for $A^{\prime}$. Then $A^{\prime}$ has a nondegenerate realization. One obviously may assume that it lies in the $(m-1)$-sphere $S^{m-1} \subset S^{n-1}$ determined by the points $e_{1}, \ldots, e_{m}$ and that the first $m-1$ points of the realization are $a_{1}, a_{2}, \ldots, a_{m-1}$. Denote by $b$ the last point of this realization. Then

$$
b \in \partial B \text {. }
$$

As the point $b$ rotates in $S^{m-1}$ about $S^{m-3}$ (determined by $a_{1} \cdots a_{m-2}$ ), it travels a circumference $C$ (nondegenerate since $b \notin S^{m-3}$ ), see Figure 1 . The 2-sphere $S^{2}$ determined by $C$ is orthogonal to $S^{m-3}$ and thus to $S^{m-2}$. Therefore the great circle $S^{1}=S^{2} \cap S^{m-2}$ includes a diameter $c_{0} c_{\pi}$ of the circle $K=S^{2} \cap B$, see Figure 1. (By a diameter, we mean here a longest geodesic in $K$ that can be longer than $\pi$ when the radius $\alpha_{m-1 m}^{\prime}$ of $B$ is $>\pi / 2$.) Due to (27),

$$
b \in C \cap \partial K .
$$

Note that the points $b_{0}$ and $b_{\pi}$ which make up $C \cap S^{1}=C \cap S^{m-2}$ satisfy

$$
a_{i} b_{0}=a_{i} b_{\pi}=\alpha_{i m}^{\prime}, \quad i=1,2, \ldots, m-2 .
$$

Thus each of them is either in $F_{0}$ or $F_{\pi}$; however, if one is in $F_{0}$ then the other one should be in $F_{\pi}$ since $b_{0}$ and $b_{\pi}$ are distinct and symmetric about $S^{m-3}$. One may assume that

$$
b_{0} \in F_{0} ; \quad b_{\pi} \in F_{\pi} .
$$

By contrary assumption (25), $b_{0}$ and $b_{\pi}$ lie in the open ball $B$. Therefore $b_{0}$ and $b_{\pi}$ are interior points of the diameter $c_{0} c_{\pi}$, see Figure 1. Obviously the circumference $C$ is orthogonal to $c_{0} c_{\pi}$ at $b_{0}$ and $b_{\pi}$. Then $C$ and $\partial K$ cannot intersect contrary to (28). The case $F_{0} \cup F_{\pi} \subset S^{m-1} \backslash(B \cup G)$ reduces to a contradiction, similarly, which proves $(24)$.

H. Since the points $b_{0}, b_{\pi} \in S^{m-2}$ are symmetric about $S^{m-3}$ and (see (30)) $b_{0} \in F_{0}$ thus lying on the same side of $S^{m-3}$ with $a_{m-1}$, the distance

$$
b_{0} a_{m-1} \leq b_{\pi} a_{m-1} \text {. }
$$

Now if $F_{\pi} \subset B$ and, by (24), $F_{0} \subset S^{m-1} \backslash(G \cup B)$ then by (30) the distance $b_{\pi} a_{m-1}<b_{0} a_{m-1}$ contrary to (31). Hence

$$
F_{0} \subset B, \quad F_{\pi} \subset S^{m-1} \backslash(G \cup B) .
$$

Now come back to the distance $\alpha(\phi)=a_{m-1} a(\phi)$ singled out in subsection D. Due to (32) and (21), one has

$$
\alpha(0)<\alpha_{m-1 m}^{\prime} \leq \alpha_{m-1 m}^{\prime \prime}<\alpha(\pi)
$$

I. Now we watch the distance $p_{m-1} p(\phi)$ in $M^{n}$. For the triangle $p_{m-1} p_{0} p(\phi)$, consider in the $k^{\prime}$-plane $\left(k^{\prime \prime}\right.$-plane) a triangle with the same side lengths. Denote by $\alpha^{\prime}(\phi)\left(\alpha^{\prime \prime}(\phi)\right)$ its angle opposite to the side of the length $p_{m-1} p(\phi)$. Due to (12) and (15),

$$
\alpha^{\prime}(\phi) \leq \alpha(\phi) \leq \alpha^{\prime \prime}(\phi) .
$$

Suppose now that $p_{m-1} p(0) \geq l_{m-1 m}$. Due to (34) and geometry of the $k$-plane, one has then $\alpha(0) \geq \alpha^{\prime}(0) \geq \alpha_{m-1 m}^{\prime}$ contrary to (33). Thus $p_{m-1} p(0)<l_{m-1 m}$. 
Similarly, $p_{m-1} p(\pi)>l_{m-1 m}$. By continuity, $p_{m-1} p\left(\phi^{*}\right)=l_{m-1 m}$ at some $\phi^{*} \in(0, \pi)$.

Thus an arbitrary realization $p_{0} p_{1} \cdots p_{m-1}$ of $L_{m}$ fitting the frame $\left\{e_{1}, e_{2}\right.$, $\left.\ldots, e_{m-1}\right\}$ has been augmented by the point $p_{m}=p\left(\phi^{*}\right)$ such that $p_{0} p_{1} \cdots p_{m}$ is a realization of $L$. Obviously this realization fits the frame $\left\{e_{1}, e_{2}, \ldots, e_{m}\right\}$. This completes the proof. (Again, we do not know if $\phi^{*}$ and $p_{m}$ are unique.)

\section{REFERENCES}

1. A. D. Alexandrow, Über eine Veralgemeinerung der Riemannschen Geometrie, Schriftenreihe Inst. Math. Deuts. Acad. Wiss. I (1957), 33-84.

2. Yu. D. Burago, and V. A. Zalgaller, Convex sets in Riemannian spaces of non-negative curvature, Russian Math. Surveys 32 (1977), 1-57.

3. B. V. Dekster and J. B. Wilker, Simplexes in spaces of constant curvature, Geom. Dedicata 38 (1991), 1-12.

4. D. Gromoll, W. Klingenberg, and W. Meyer, Riemannsche Geometrie im Grossen, Springer Verlag, Berlin, Heidelberg, and New York, 1968.

Department of Mathematics, Mount Allison University, Sackville, New Brunswick, CANADA E0A 3C0

E-mail address: MBARGIEL@MTA.CA 